\title{
Genetic Learning: A New Conceptual Framework for Studying the Impact of the Environment on Changes in Genetic Behavior
}

\author{
Sean Hughes \& Jan De Houwer
}

\begin{abstract}
Research shows that genetic material and environment are engaged in an ongoing conversation, one that dictates both physiology and psychology. In this paper we argue that genetic material and environment are not engaged in a single conversation but rather multiple types of conversations, and offer researchers a set of conceptual tools to better understand what nature and nurture are saying to one another. In Part I we highlight three types of activity that can be studied: behavior, changes in behavior, and learning. We define what it means to say that learning has taken place and highlight different ways in which living organisms do so. In Part II we apply this analysis to the genetic level. We argue that until now, the life and social sciences have primarily focused on two types of genetic activity (genetic behavior and changes in genetic behavior), while a third (genetic learning) is still waiting to be explored systematically. In Part III we consider how our approach better organizes past, present, and future work on genetic activity (heuristic value) and opens up a new research area centered on genetic learning (generative value).
\end{abstract}

Over the past decades, it has become increasingly clear that many stimuli in our daily lives, from alcohol and tobacco, to environmental pollutants, and psychological stressors, can alter how genetic material is regulated and expressed (see [1], [2], for book length treatments). Other events such as diet and nutrition [3] [4], trauma [5], parenting behaviors [6], and adverse social environments [7] also trigger profound and long-lasting changes in genetic activity. Such work indicates that genetic material is capable of reacting to events in the environment.

In this paper we ask a different question: can genetic material learn? Before we can answer that question we first need to clarify what learning is and when it is said to take place. In Part I, we highlight a definition of learning that is not only precise but that can also be applied in many contexts and in many systems. This definition helps us to appreciate that there are three different types of activity we can study: behavior, changes in behavior, and learning. We briefly consider each and how they relate to one another. Later, in Part II, we introduce a definition of genetic learning and describe the ways in which genetic material can be said to learn. Doing so will help distinguish the study of genetic behavior from the study of genetic learning. Finally, in Part III, we consider the merits of our approach: it helps us to organize and understand previous work on genetic activity (heuristic value), and introduces many new questions about learning at the genetic level (generative value). Our approach highlights that previous work on the interaction between environment and genetic material has been relatively "static" (i.e., focused on how single environmental events impact genetic material) and highlights opportunities for studying the dynamic nature of this interaction (i.e., focusing on how the impact of environmental events on genetic material changes over time).

Before we begin, we would like to highlight two points. First, our main aim in this paper is to make a conceptual contribution that changes the way we think and talk about the relationship between genetic material and environment. We believe that knowledge about learning in one system (whole organisms) may help us develop and expand our knowledge about learning in other systems (genetic system). Second, the definitions we introduce are not 'correct' or 'true' in some absolute sense (i.e., they are not ontological claims). It is difficult to arrive at universal agreement on what is the "true" or "best" definition of any phenomenon and we do not want to get side-tracked into such a debate. Rather our aim is to provide 'useful' definitions, more specifically, definitions that allow us to predict and influence behavior in all kinds of systems, including genetic material. 
Part I: Behavior, Changes in Behavior, \& Learning

What is Behavior?

Certain words and concepts are so woven into the fabric of everyday language that we rarely stop and consider what they actually mean. For instance, if we were to ask a person on the street to define terms such as 'gravity', 'speed', and 'force', most would probably give examples of these phenomena rather than the definitions we were looking for. The same holds for 'behavior'. If asked to define behavior we may be tempted to say that it is 'what living organisms do' or offer examples of our own actions that spring to mind. In this paper we go one step further and define behavior as 'a transition in the state of a system that is due to a stimulus'. This definition is comprised of several components that may be new to many readers. So let's consider each in turn.

First, we have to specify the object whose behavior we are interested in. We refer to this object as a 'system'. This term is purposefully abstract because the system under investigation will ultimately depend on the scientist's goals or objectives. For some (e.g., psychologists) the system is a 'whole organism' (e.g., a person), for others (e.g., neuroscientists, geneticists) it is a constituent part of an organism (e.g., neuron, genetic material). For still others (e.g., anthropology, sociology) the system may be entire collections of organisms (e.g., herds, colonies, societies) or even a nonliving object, such as a robot (computer science), river (geology), or black hole (astronomy). By emphasizing the system we are studying we orient ourselves and others to a specific subset of behaviors - namely - those that can be elicited or emitted by that particular system.

Second, we use the term 'state' to highlight that the system is observed to occupy some position along a given dimension at a certain moment in time. For instance, a living organism (e.g., a person) might be calm or panicking. A component of an organism (e.g., a DNA segment) may be expressed at a high or low rate. Likewise, a cleaning robot may be motionless or moving. Third, the system under investigation must be capable of variation along some dimension. We refer to this as a 'transition in state'. For instance, a person may be observed to transition from being calm to panicking; a DNA segment from being expressed at a low to high rate, and so on. In some cases this transition may be along the same dimension (e.g., low to high levels of panicking) and in other cases it may be along different dimensions (from panicking to laughing). ${ }^{1}$

Fourth, a transition in state is typically due to something in the environment, that is, it would not have occurred without some element in the environment. We refer to this element in the environment as a 'stimulus'. Our definition of a stimulus is abstract and orients us towards the idea that an environmental event can lead to a transition in state. A loud bang (stimulus) may lead a person to transition from one state (being calm) to another (panicking). The presence of a physical impediment such as a wall (stimulus) may lead a cleaning robot to transition from moving in one direction to another. Similarly, exposure to an environmental pollutant (stimulus) may lead to differences in the rate of expression of certain DNA segments (i.e., a transition from one state to another in the system). ${ }^{2}$

When we combine these various elements together we arrive at our definition of behavior: a transition in the state of a system that is due a stimulus in the environment. In the above examples, the transition from being calm to panicking was due to a loud bang whereas a DNA segment's transition from a low to high rate of expression was due to the presence of an environmental pollutant. Thus the term behavior is explanatory: it orientates us towards the fact that a system has transitioned from one state to another, and tells us something about why that transition has occurred (i.e., it is due to a stimulus insofar as it would not have occurred had that stimulus been absent; see Figure 1). ${ }^{3}$

\section{What is a Change in Behavior?}

Most systems are not only capable of behaving (i.e., of transitioning from one state to another because of a stimulus) but also of demonstrating changes in behavior. To illustrate, consider the grip reflex present in newborn babies (see [8]). Stroking the palm of an infant (system) with one's finger (stimulus) when the

\footnotetext{
${ }^{1}$ In reality, most systems will be capable of variation within and between multiple dimensions. It is also worth noting that there is merit in thinking about transitions between states in a probabilistic manner (e.g., there is a higher or lower probability of the system being observed to transition from one state to another at a given moment in time). For ease of communication, however, we will talk about transitions that do or do not occur at a given moment in time.

${ }^{2}$ It's worth repeating that we are not making any ontological claims here. We simply believe that it can be useful to formulate assumptions about the presence of events in the world and about the functions of those events (e.g., their function to alter the state of a system). Whether a stimulus has a particular function (e.g., whether it is actually responsible for the transition in the state of a system) can be established by manipulating the presence of the stimulus and observing how this influences the system. Many events can function as a stimulus, from the presence of an event (e.g., a loud bang), and absence of an event (e.g., absence of a loud bang), to time itself (e.g., duration since a loud bang was previously encountered).

${ }^{3}$ Note that behavior refers to more than a mere transition in state: it refers to a transition in state that is due to a specific stimulus. We view the terms 'response' and 'behavior' as synonyms: they are both explanations in that they attribute a transition in state to a specific cause (a stimulus). Although the term 'response' highlights this point nicely (i.e., one 'responds' to something) the term 'behavior' may be more familiar to readers and will therefore be used through the remainder of this paper.
} 


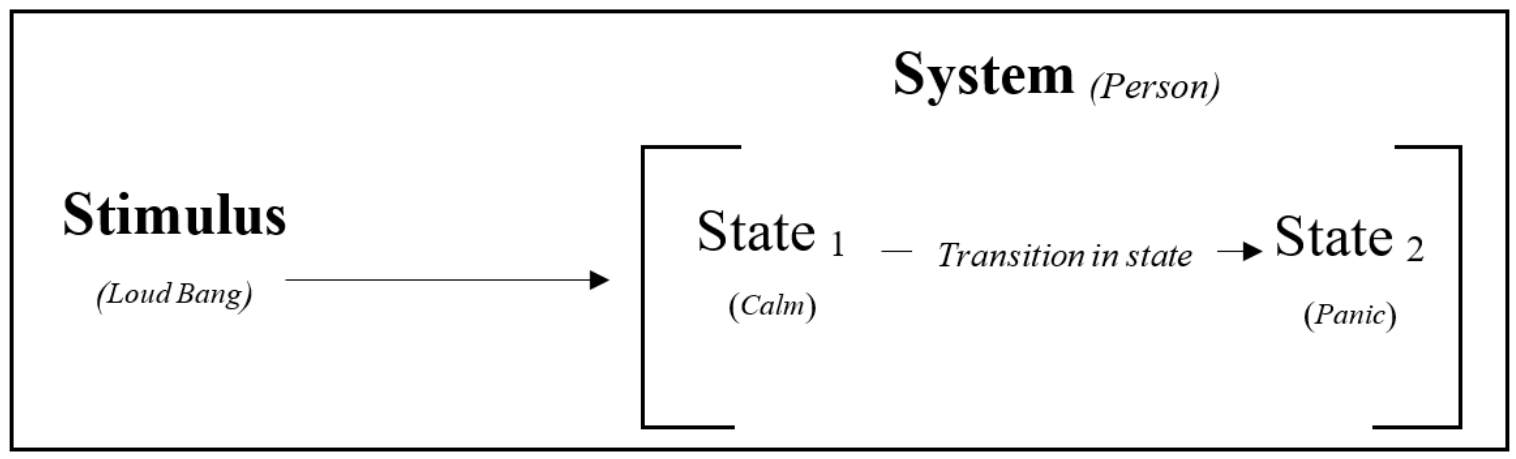

Figure 1. A visual illustration of behavior (i.e., a transition in the state of a system due to a stimulus). The system is the object whose behavior we are interested in (e.g., a person). A state refers to some position along a given dimension at a certain moment in time (e.g., being calm). A transition in state refers to a change from one state to another (e.g., from being calm to panicking). A stimulus is something in the environment that can lead to a transition in state (e.g., a loud bang). Behavior refers to a transition in state due to a stimulus (e.g., from being calm to panicking after hearing a loud bang).

infant is one month old will lead it to transition from one state (its hand being open) to another (its hand being shut and gripping the finger). Yet stroking the same palm when it is six months old is unlikely to produce the same transition in state (e.g., the hand now stays open). The presence of the grip reflex at Time 1 constitutes a behavior (i.e., it is a transition in state due to a stimulus), while its absence at Time 2 reveals that behavior has changed (i.e., stroking the palm leads to a transition in state at Time 1 but no longer has that effect at Time 2). In this way we see that a change in behavior refers to a difference, namely, a difference in the way that the same stimulus influences the state of the system at Time 1 versus Time 2 . The term 'change in behavior' is therefore descriptive: it simply highlights that a system's activity has changed. Explaining why that change has occurred brings us to the topic of learning.

\section{What is Learning?}

Learning can be defined in many ways (e.g., [9], [10]). In this paper we define it as a change in behavior that is due to a regularity in the environment (see [11]). This definition has proven useful when studying whole living organisms (see [8] for an overview) and we believe that it can be applied to other systems as well. According to this definition, when we study learning we are doing two things: (a) acknowledging that the impact of a stimulus on the state of a system has changed, and (b) explaining why this change has occurred through reference to an environmental "regularity". The concept of a 'regularity' as we define it may be new to some, but it's simply a term denoting any event "in the environment...that involves more than the presence of a single stimulus or behavior at a single moment in time" [11]. There are three main types of regularities. First, a single stimulus can be presented repeatedly (e.g., the repeated presence of a loud bang). Second, two stimuli can be presented together once or multiple times (e.g., a bell that precedes food). Third, a stimulus might follow a behavior (e.g., a food pellet is presented after a rat presses a lever). The impact of a regularity on behavior can be established by comparing behavior at a time before the regularity has been experienced (Time 1) with behavior after the regularity has been experienced (Time 2). If behavior at Time1 (e.g., a loud bang evokes a strong response) differs from behavior at Time 2 (e.g., the same loud bang evokes only a weak response), then it is likely that this change in behavior is due to the regularity that occurred between Time 1 and Time 2 (e.g., the repeated presentations of the loud bang). If this is the case, learning has taken place. Different types of learning vary with regard to the type of regularity that causes the change in behavior.

\section{Three Different Types of Learning}

Learning via a Regularity Involving One Stimulus (e.g., Habituation \& Sensitization)

The most basic form of learning involves a change in behavior that is due to the repeated presentation of one stimulus. To illustrate, imagine that you are on a quiet street and the exhaust of a passing car backfires. The first time you hear this loud bang (stimulus) may cause you to transition from being calm to highly startled. Now imagine that you are walking down the same street later that day, the same car passes by, and its exhaust once again backfires. This time you may be startled to a far lesser extent than before (i.e., show a different transition in state to the same stimulus). This reduction in the intensity of the startle response from Time 1 to 2 constitutes a change in behavior. This change in behavior is due to a regularity - namely - the repeated occurrence of a single stimulus (i.e., the loud bang) between Time 1 and Time 2. Hence, learning is said to have occurred. Common examples of this type of learning are habituation (what we have just described; see Figure 2) or sensitization (an increase in the intensity of a response [e.g., frustration] due to the repeated presentation of a stimulus [e.g., sound of construction work going on outside your office window]). 
Time 1: Behavior ${ }_{1}$ (Being Intensely Startled)

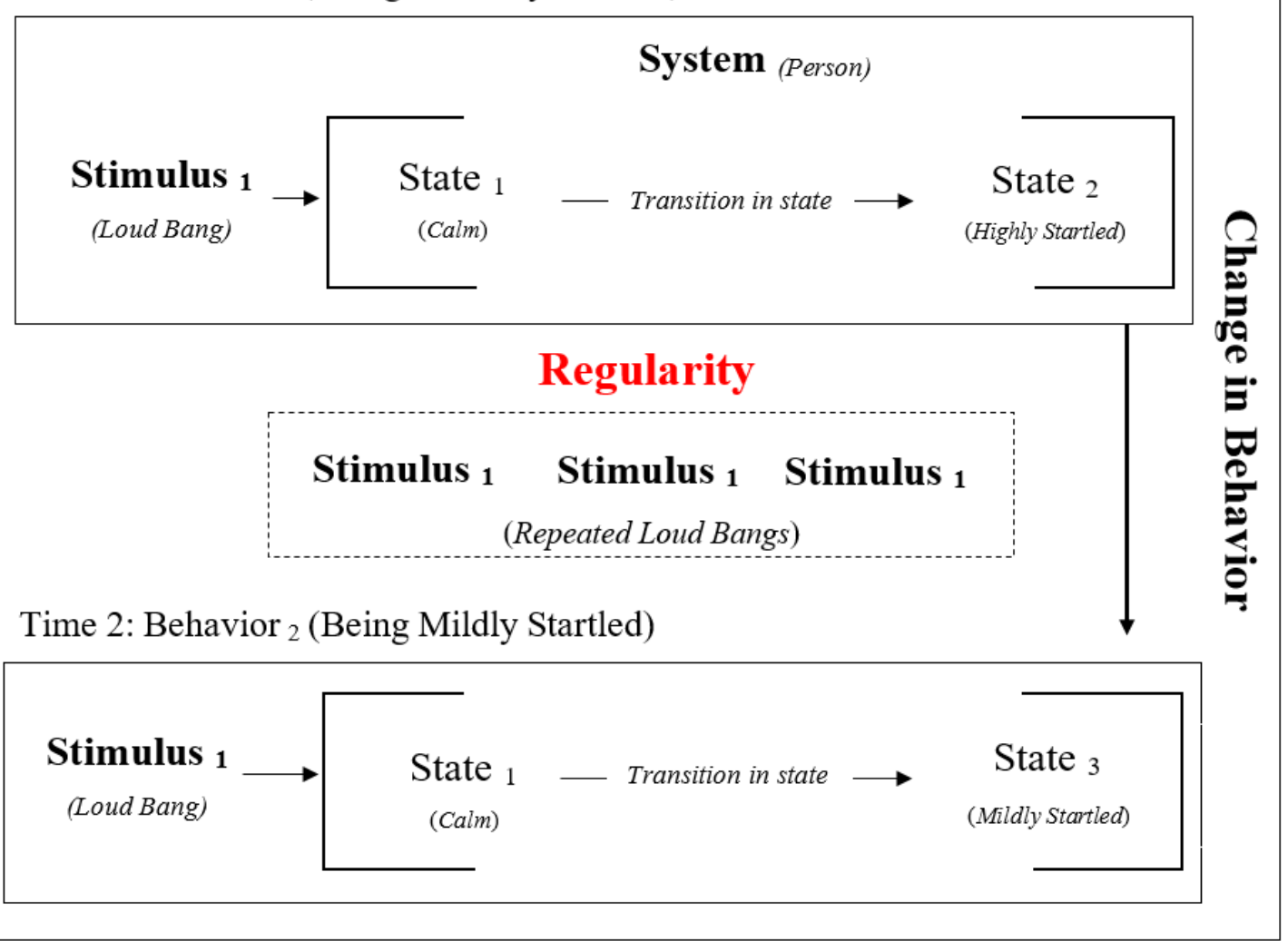

Figure 2. A visual illustration of a change in behavior due to a regularity in the presence of one stimulus. A loud bang (Stimulus ${ }_{1}$ ) initially leads to a transition in state (e.g., from being calm to highly startled). Later on the same stimulus (loud bang) leads to a different transition in state (e.g., from being calm to mildly startled). This difference between the first and second transitions represents a change in behavior. This change in behavior is due to a regularity - namely - the repeated occurrence of the loud bang between Time 1 and Time 2. This represents one instance of learning (i.e., a habituation effect).

Learning via a Regularity Involving Two Stimuli (e.g., Classical Conditioning)

A second type of learning involves a change in behavior that is due to a regularity involving two stimuli. This type of learning is known as classical conditioning (see [8], for an overview). To illustrate, imagine that we ring a bell (Stimulus 1 ) in the presence of a dog (system). At Time 1, the bell does not lead to the transition in state we are interested in (salivation). Later on we introduce a regularity involving two stimuli: we pair the ringing of the bell with the administration of food. At Time 2, the bell (Stimulus 1) now leads to the transition in state we are interested in (salivation). This increase in salivation from Time 1 to 2 constitutes a change in behavior and this change is due to a regularity between the ringing of the bell and food. In this case we can say that learning has taken place and refer to it as a classical conditioning effect (see Figure 3). ${ }^{4}$
Learning via a Regularity Involving Stimuli \& Responses (e.g., Operant Conditioning)

A third type of learning involves a change in behavior that is due to a regularity involving stimuli and responses. This is often known as operant conditioning. To illustrate, imagine that we place a rat (system) into a training chamber which contains a light, a lever which can be pressed, and a food dispenser. At Time 1, turning on the light in the chamber does not lead to the transition in state we are interested in (i.e., to lever pressing). We then introduce a regularity such that if - in the presence of the light (Stimulus 1) - a transition does occur from one state (not pressing the lever) to another (pressing the lever; e.g., because the rat is prompted into pressing the lever) then food is delivered (Stimulus 2). At Time 2, we now observe that the presentation of the light results in the transition in state we are interested in (lever pressing). This increase in lever pressing from Time 1 to 2 constitutes a change in behavior and this change is due

\footnotetext{
${ }^{4}$ Two points are worth noting about Figure 3. First, although the system (dog) is not behaving in the way we are interested in at Time 1 (salivating in the presence of a ringing bell) it is likely behaving in many other ways (e.g., growling, exploring, and breathing). Thus the absence of behavior at Time 1 does not mean that the system is not behaving per se. It is just not behaving in the way we are ultimately interested in (i.e., it is not salivating). Second, in order to study any type of learning we must first know what system, states, transitions, and stimuli we are ultimately interested in (i.e., we must be clear about our scientific goals and objectives). We need to know this before we can determine if a change in behavior has taken place and if this change was due to a regularity. We will return to this point in Part II in the context of genetic learning.
} 
to a regularity involving stimuli and responses: the fact that pressing the lever (transition in state) when the light (Stimulus 1) was turned on leads to food (Stimulus 2 ). This instance of learning is often referred to as an operant conditioning effect (see Figure 4 and [8] for an overview).

Why Define Learning in this Way?

Defining learning in this way has several benefits. First, it highlights that there are three types of activity scientists can study: how a system behaves, changes its behavior, or learns. When we use the term behavior we are explaining that a transition in state has occurred and attribute that transition to a particular cause (a stimulus). When we use the term change in behavior we are describing a difference in the way the same stimulus impacts the transition in state of a system, and when we use the term learning we are explaining why that change in behavior occurred (i.e., because of an environmental regularity).
This definition not only allows us to distinguish between different types of activity but also different types of learning. Habituation, classical conditioning, and operant conditioning each represent a type of learning. But they all differ in terms of the regularity that gives rise to the change in behavior (i.e., habituation: regularity involving one stimulus; classical conditioning: regularity involving two stimuli; operant conditioning: regularity involving stimuli and responses). Defining learning in this way also leads to an interesting new idea, one that has not really been appreciated to date. Whereas past work has mainly focused on learning in one system ('whole organisms'; see [8]) many other types of systems may be capable of learning as well, systems such as neurons, cell assemblies, and even genetic material. Therefore, in Part II, we consider what it means to say that genetic material behaves and changes its behavior. We then consider if it can also learn.

\section{Learning (Regularity: two stimuli)}

Time 1: Behavior of interest (salivating) is absent

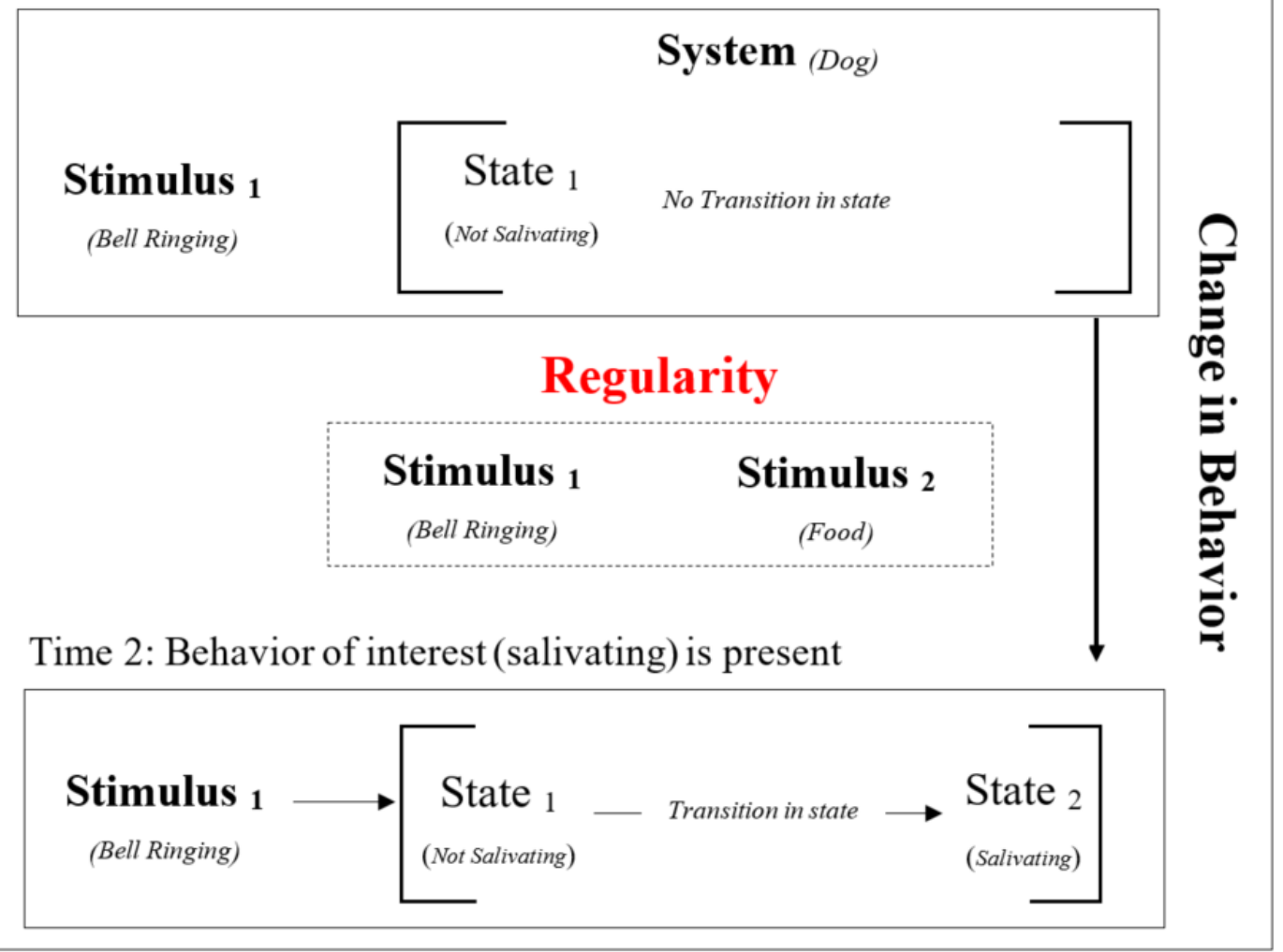

Figure 3. A visual illustration of a change in behavior due to a regularity between two stimuli. At Time 1, ringing a bell (Stimulus ${ }_{1}$ ) does not lead to the transition in state we are interested in. Ringing the bell (Stimulus ${ }_{1}$ ) is then paired with the administration of food (Stimulus 2), either once or repeatedly. At Time 2, ringing the bell (Stimulus ${ }_{1}$ ) now leads to the transition in state we are interested in (salivating). This increase in salivation from Time 1 to 2 constitutes a change in behavior and this change is due to a regularity between two stimuli (i.e., pairing the bell with food) that occurred between Time 1 and Time 2. This constitutes a second type of learning referred to as a classical conditioning effect. 
Learning (Regularity: stimulus \& response)

Time 1: Behavior of interest (lever pressing) is absent
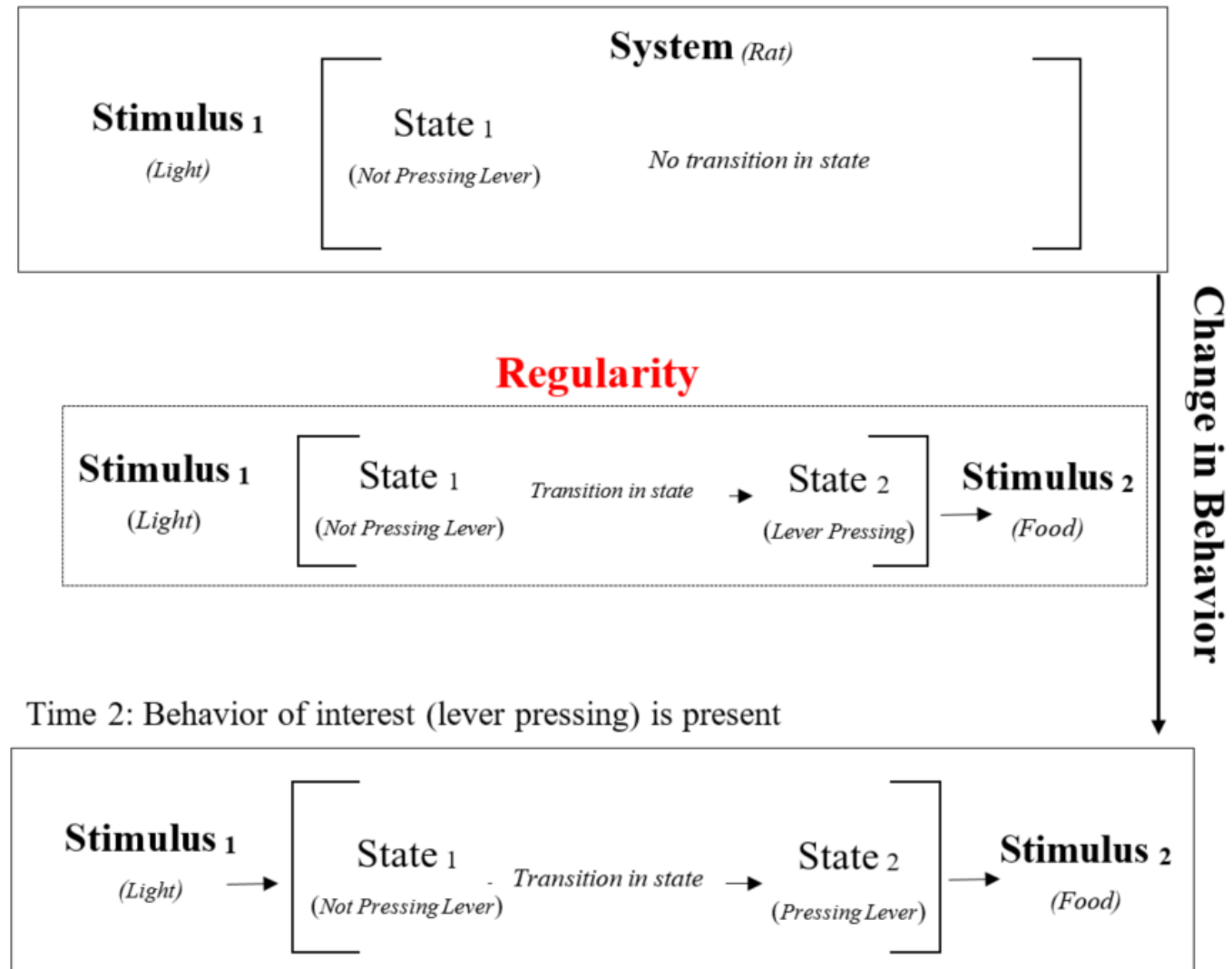

Figure 4. A visual illustration of a change in behavior due to a regularity involving stimuli and responses. At Time 1 a system (rat) does not demonstrate the transition in state we are interested in (i.e., lever pressing) in the presence of a light (Stimulus 1). A regularity is then introduced: whenever the transition in state occurs (lever pressing) in the presence of the light (Stimulus 1), it is followed by the administration of food (Stimulus 2). At Time 2, the transition in state we are interested in (lever pressing) now occurs in the presence of the light. This increase in lever pressing from Time 1 to 2 constitutes a change in behavior that is due to a regularity involving stimuli and responses (i.e., to the fact that lever pressing previously led to food in the presence of the light) that occurred between Time 1 and Time 2. This constitutes a third type of learning known as an operant conditioning effect. Note: When we say that at Time 2, the transition in state is a function of Stimulus 1 , this does not imply that the transition in state is only a function of Stimulus 1. In many cases of operant conditioning, the transition in state is also a function of Stimulus 2 (food), more specifically the fact that the transition in state is followed by a reinforcer.

Part II: Genetic Behavior, Changes in Genetic Behavior, \& Genetic Learning

What is Genetic Behavior?

A first step in our analysis is to introduce the concept of genetic behavior. We define it as a transition in the state of genetic material that is due to a stimulus in the environment. Let's consider each of these components in turn. The system whose behavior we are interested in is genetic material. When we use the term 'genetic material' we are not only referring to 'genes' (i.e., the "union of genomic sequences encoding a coherent set of potentially overlapping functional products" [12]) but all material that comprises the genetic system (i.e., those found 'around' the DNA, in the nucleus, mitochondria, and cytoplasm which play a fundamental role in genetic regulation and expression). This material may be currently embedded in human and non-human organisms, be found outside of those organisms (e.g., in-vitro cultures), or even part of a generation, an entity or species (e.g., transgenerational genetic material may change structurally at the level of the DNA, or 'around' the DNA, inducing regulatory differences throughout generations) [13]. Examples of material that surrounds and regulates the DNA are epigenetic components, including methyl groups located at CpG base pairs of DNA, non-coding RNAs, histone proteins, and enzymes. Hence, all these materials are part of 'genetic material'.

We also adopt a broad perspective on the concept of 'stimulus' and 'environment'. The 'environment' is defined as the context in which genetic behavior takes place and can refer to a context inside of the body (e.g., intra- or extracellular context, presence of hormones, the uterine environment during pregnancy) or outside of the body (e.g., physical and social settings of the organism within which the genetic material is embedded, the laboratory culture within which the 
genetic material is being cultivated). In other words, environments can vary from the micro (e.g., molecular settings) to molar (e.g., the social or cultural context in which genetic material is embedded). Stimuli are events in the environment that can change the state of systems in that environment.

How we define stimulus and environment will ultimately depend on our scientific goals, and whether doing so in a certain way helps us to achieve those goals. For instance, if we were interested in how socioeconomic status or maternal deprivation (stimulus) influence transitions in the state of genetic material then we would focus on a different type of environment and set of stimuli (e.g., social, geographical, and cultural practices) than if we were interested in how injecting a chemical into the cell (stimulus) influences those same transitions.

One might also ask what qualifies as a transition in state at the genetic level. Does it involve a transition in the state of the entire genome, or perhaps the activity of a group of genes, or even a single gene? Unsurprisingly we take a broad view here too. Recall that the term 'state' highlights that the system is observed to occupy some position along a given dimension at a certain moment in time, while a 'transition in state' refers to variation in states along the same or different dimensions. What states and transitions qualify will once again depend on our scientific goals and objectives. If the goal is to see how a chromosome transitions from one state to another as a function of a certain stimulus (e.g., poor diet and nutrition) then states and transitions will be defined broadly in terms of the entire chromosome. If the goal is to transition the state of a single gene (e.g., from higher to lower rates of expressing for a specific protein) through exposure to a stimulus (e.g., chemical) then states and transitions will be far narrower. The key message here is that state(s) and transitions of genetic material can vary in their scope and nature. Which are examined will depend on what it is we are trying to study, and whether defining them in a given way helps us to achieve that goal.

Combining the above concepts leads to our definition of genetic behavior: a transition in state of genetic material that is due a stimulus in the environment. Two points are worth noting here. First, before we can go about studying genetic behavior we need to know what our scientific goal is. Only then can we orient ourselves towards the specific system, states, transitions, stimuli, and environments that comprise the genetic behavior of interest. Second, genetic behavior is not simply a description but rather an explanation: it highlights that genetic material has transitioned from one state to another, and tells us something about why that transition has occurred (i.e., because of a stimulus in the environment). For instance, exposure to acute food deprivation (stimulus) leads to an increase in lipolysis gene expression (transition in state) in the liver of rats (system) [14]. Similarly, shortterm exposure to certain air pollutants (stimulus) is associated with an increase in HSPA8 gene expression levels (transition in state) in humans (system) [15].

The above examples show that genetic behavior is not just a description of a transition in state of genetic material. Rather it is an explanation as to why that transition occurred (because of a stimulus in the environment, like food deprivation or pollution). Our perspective on genetic behavior is therefore contextual and relational: it acknowledges the interdependence and co-determination between genetic material and the environment in which it is embedded (for a similar view see $\left.{ }^{[16]}\right)$. Genetic behavior as defined in this way has been studied extensively by geneticists. In contrast, less attention has been given to what we call changes in genetic behavior. ${ }^{5}$

What is a Change in Genetic Behavior?

The ability for genetic material to behave does not immediately imply that it can also change its behavior. In order to speak of changes in genetic behavior a second condition must be met. Specifically, a change in genetic behavior requires that there is a difference in the impact that the same stimulus has on the state of genetic material. To illustrate, imagine that we take a newly born rat during its first week of life, introduce a stimulus into its environment (e.g., separate it from its mother for 30 minutes) and assess for a transition in the state of its genetic material. When we do so we notice that a transition in state takes place (e.g., there is an increase in gene product c-Fos expression in the hypothalamus and limbic forebrain). Two weeks later we expose the rat pup to maternal separation once again and investigate if the same transition in state occurs. This time, however, we notice a different transition in state (e.g., gene product c-Fos is expressed at even higher levels than before) (for more on this type of work see [17]).

In our hypothetical example, we observe a genetic behavior at Time 1: initial exposure to a stimulus

\footnotetext{
${ }^{5}$ Researchers can study genetic learning in two ways: either from a functional perspective that seeks to understand the environmental events that moderate genetic learning (i.e., that determine to which extent environmental regularities cause changes in genetic behavior), or from a mechanistic perspective that seeks to identify the (epi)genetic mechanisms that mediate genetic learning (i.e., the mechanisms via which environmental regularities causes changes in genetic behavior). The current paper is focused on the functional level. Although we do not unpack the mechanistic underpinnings of genetic learning (for examples of such work see [26-28]), our ideas are designed to serve as inspiration for future mechanistic research (e.g., do the same or different biological mechanisms mediate different types of genetic learning [sensitization, classical- and operant conditioning])? Do the same mechanisms mediate genetic learning in different organisms or species? How, when, and why are those mechanisms impacted by different moderators in the environment?).
} 
(maternal separation) led to one transition in state (e.g., a small increase in c-Fos expression) in genetic material (system). We also observe a genetic behavior at Time 2: exposure to the same stimulus two weeks later now leads to a different transition in state (e.g., a large increase in c-Fos expression). This difference in rate of c-Fos expression from Time 1 to 2 would represent a change in the impact of the same stimulus (maternal separation) on the state of genetic material and would therefore represent a change in genetic behavior. 6

What the above example hopefully makes clear is that a 'change in genetic behavior' is a descriptive term used to highlight that the genetic system's activity has changed in some way. Explaining why this change has occurred brings us to the topic of genetic learning.

\section{What is Genetic Learning?}

Genetic learning is defined as 'a change in genetic behavior that is due to a regularity in the environment'. Hence, in order to conclude that genetic learning has taken place, one not only has to observe that behavior at Time 1 is different from behavior at Time 2 but also establish that the change in behavior is due to a regularity (i.e., that the change in behavior would not have occurred in the absence of the regularity). For instance, if maternal separation leads to more c-Fos expression at Time 2 then at Time 1 but only if the rat repeatedly experienced maternal separation between Time 1 and Time 2, then it is reasonable to conclude that the increase in c-Fos expression from Time 1 to Time 2 is due to a regularity, more specifically, the repeated experience of maternal separation. In this case, genetic learning has taken place. Different types of genetic learning can be distinguished on the basis of the type of regularity that causes the change in genetic behavior. Note that much of what we propose remains hypothetical in nature: demonstrations of certain genetic learning effects still await empirical scrutiny.
Three Different Types of Genetic Learning

Genetic Learning via a Regularity Involving One Stimulus (Genetic Habituation and Sensitization)

The most basic form of genetic learning involves a change in genetic behavior that is due to the repeated presentation of one stimulus. To illustrate, consider the idea of 'stress memory' in plants. When certain varieties of maize plants are subjected to the same stimulus (dehydration) over and over again they show improved retention of water compared to the first time they encountered that stimulus (e.g., [19] [20]). These changes in behavior in the face of repeated contact with the stimulus are not only evident at the organismic and cellular levels, but the genetic as well: transcription levels of stress response genes are significantly higher after multiple presentations of a stressful stimulus (dehydration) compared to the first presentation of that stimulus (for more on 'transcriptional stress memory' see $[21])$.

According to our perspective, the transition in state (low transcription levels of stress response genes) produced by the stimulus (dehydration) at Time 1 is an example of a genetic behavior. So too is the transition in state (high transcription levels) that occurs when the same stimulus (dehydration) is presented at Time 2 . This increase in transcription levels from Time 1 to 2 constitutes a change in genetic behavior and this change appears to be due to the occurrence of the same stimulus (dehydration) over and over again. Genetic learning can therefore be said to take place, and in this case, represents an instance of genetic sensitization (see Figure 5). If the intensity of gene expression were to decrease as a function of repeated stimulus presentations then we would instead speak of genetic habituation. ${ }^{7}$

\footnotetext{
${ }^{6}$ It is worth noting that work in this area has tended to expose a rat pup to either a single instance of maternal separation or to multiple instances of maternal separation (see [18]). From our perspective, this only allows one to compare two types of behavior: responses to single instance of maternal separation and the response to multiple instances of maternal separation. Yet, in order to conclude that a change in genetic behavior has taken place, one needs to observe a change in the impact of the same stimulus on the state of genetic material. For instance, if the response to a single instance of maternal separation is different at Time 1 than at Time 2, one can say that behavior (i.e., the response to a single instance of maternal separation) has changed. As we discuss below, the conclusion that genetic learning has taken place requires still one additional element, namely the assumption that the change in behavior is due to a regularity (e.g., is due to the repeated experience of maternal separation between Time 1 and Time 2).

7 The role of royal jelly in the development of queen bees also seems like a candidate for this type of learning (see [22] [23] [24]). A change in behavior (decrease in DNMT3 expression - and the associated upregulation of developmental genes in queen-destined larvae) appears to take place when the system is exposed to the same stimulus over and over again (royal jelly). Note that testing this claim would require that we systematically manipulate the number of times different instances of a system (honey bee larvae) are exposed to the same stimulus (jelly) while controlling for possible confounding factors (e.g., mere passage of time) and that we look for corresponding differences in their genetic behaviors.
} 


\section{Genetic Learning (Regularity: single stimulus)}

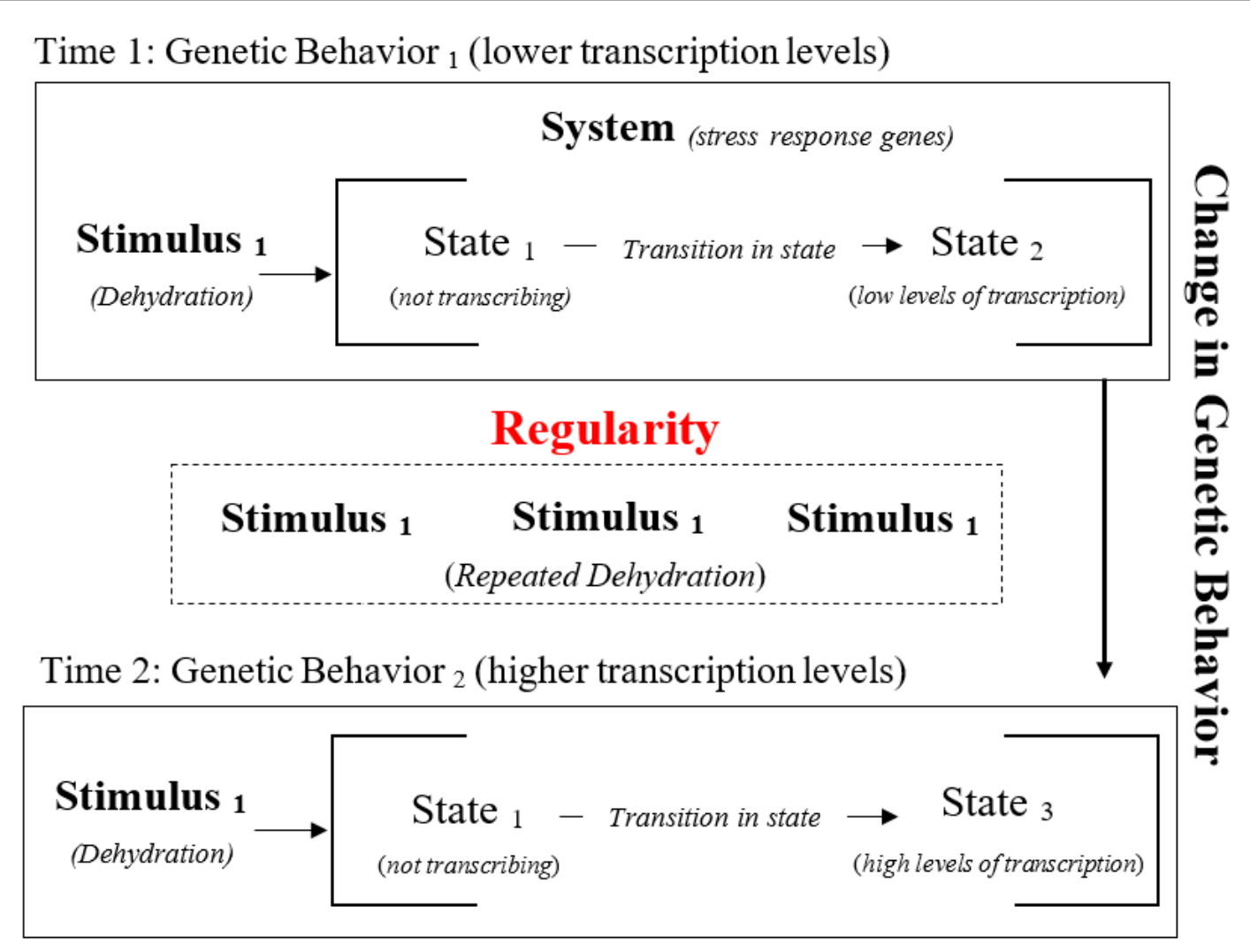

Figure 5. A visual illustration of a change in genetic behavior that is due to a regularity involving one stimulus. At Time 1, dehydration (Stimulus 1) leads to one transition in state (e.g., from no to low transcription levels of stress response genes). At Time 2, the same stimulus (dehydration) leads to a different transition in state (e.g., from no to high transcription levels of stress response genes). This increase in transcription levels from Time 1 to 2 constitutes a change in behavior and this change is due to a regularity - namely - the repeated occurrence of a single stimulus (dehydration) between Time 1 and Time 2. This represents one type of genetic learning (i.e., a genetic sensitization effect).

Genetic Learning via a Regularity Involving Two Stimuli (Genetic Classical Conditioning)

A second type of genetic learning involves a change in genetic behavior that is due to the pairing of stimuli. This can be described as genetic classical conditioning. Imagine that, at Time 1, we place a rat into a training chamber, present an auditory tone (Stimulus 1) and this fails to produce the transition in state that we are interested in (an increased expression of isoform IV of the brain-derived neurotrophic factor [bdnf] gene [system]). Later on we introduce a regularity between two stimuli (i.e., we pair the tone with the repeated administration of electrical shocks). At Time 2, exposure to the same stimulus (tone) now leads to a different transition in states (e.g., an increase in mRNA expression of isoform IV). This increase from Time 1 to 2 in mRNA expression of isoform IV would constitute a change in the bdnf gene's behavior. In this case the change in behavior is likely to be due to a regularity namely - the pairing of the tone and electric shocks. If this were to occur then we could say that genetic learning has taken place and refer to it as a genetic classical conditioning effect (see Figure 6).
Several studies have utilized fear conditioning tasks to study genetic behavior (e.g., [25] [26]; also see [27] [28] [29]). Typically they take three groups of rats and expose them to different types of procedures. The first two groups are placed in an experimental chamber and either shocked (chamber + shock) or not shocked (chamber only). The third group is also shocked but in an entirely different context (another chamber + shock). Following these experiences various transitions in state of the rat's genetic material are assessed. Results show that the probability of certain transitions in state (e.g., mRNA expression of isoform IV of the bdnf gene) are higher for the rats who were exposed to a regularity (chamber + shocks) than those exposed to just the chamber or an electric shock.

Although these studies offer tentative suggestions that genetic classical conditioning may be possible, from the perspective of genetic learning, their design is not ideal. What many studies report is a transition in the state of genetic material due to a stimulus (e.g., an increase in gene expression in a rat who was shocked relative to a rat that was not shocked [control]). Although such a finding shows that gene material can 
behave (i.e., transition from one state to another due to a stimulus) it does not speak to the issue of whether genetic material can be classically conditioned (i.e., change its behavior as a result of stimulus pairings). Strong evidence for the latter requires an experiment similar to that outlined in Figure 6. Here we would show that a stimulus (e.g., a tone) initially influences the state of genetic material in one way, and that due to the pairing of stimuli (e.g., tone paired with shocks), the same stimulus (tone) later influences the state of genetic material in a different way. In other words, we need to demonstrate that the change in behavior we are observing is stimulus specific (i.e., that there is a change in the response to the stimulus that is part of the regularity). It is unclear from previous work if this is actually the case (e.g., to what extent isoform IV of bdnf is expressed in one way when the tone is initially presented and differently after that tone has been repeated paired with shocks).

Genetic Learning via a Regularity Involving Stimuli \& Responses (Genetic Operant Conditioning)

A third type of genetic learning involves changes in genetic behavior that are due to a regularity involving stimuli and responses. This would constitute an instance of genetic operant conditioning. To illustrate, imagine that at Time 1 you take a rat, place it into a training chamber (Stimulus 1), and notice that doing so leads to a certain transition in state (e.g., from low to high expression of the CNR1 gene). Now imagine that each time this gene is expressed inside the training chamber an electric shock (Stimulus 2) is delivered. When the rat is subsequently placed in that same chamber (Stimulus 1) at Time 2, expression of the CNR1 gene now remains low. This decrease in the rate of CNR1 expression from Time 1 to 2 would constitute a change in behavior and this change is probably due to a regularity between a stimulus and response: between Time 1 and Time 2, expressing the CNR1 gene inside the training chamber previously led to the delivery of an electric shock. Such an outcome would represent evidence for genetic operant conditioning (see Figure 7).

Several genetic studies on drug use, addiction, and obesity have made use of operant conditioning procedures (e.g., [30] [31]). In some cases a whole organism (rat) is exposed to operant conditioning and a change in behavior (e.g., increase in lever pressing) is observed whenever a regularity is established between a response (e.g., lever pressing) and a stimulus (e.g., delivery of food). Often these studies also investigate whether the genetic behavior of rats who were exposed to this procedure differs from rats who were not exposed to it, and in certain cases, differences in genetic behavior are observed (e.g., in histone H3 acetylation expression) [32]. In still other studies, performance on an operant procedure is used to select out systems that differ from one another (e.g., "risk seeking" vs. "risk averse" rats) so that their epigenetic differences can be compared (e.g., [33]). Although the above studies tentatively suggest that genetic operant conditioning may be possible, strong evidence for this claim is still lacking. What is instead needed is a study that demonstrates that genetic behavior changes as a function of its consequences: that the probability of genetic behavior can be increased through the delivery of certain stimuli and decreased through the provision of others. We will return to this idea in Part III. ${ }^{8}$

\section{Conclusion}

Our definition of genetic learning has several benefits. First, it highlights that there are three types of genetic activity: genetic behavior, changes in genetic behavior, or genetic learning. Second, our definition allows one to distinguish between different types of genetic learning. Genetic habituation, classical conditioning, and operant conditioning all qualify as instances of genetic learning but differ in the regularity that leads to the change in behavior. Although past work has made reference to learning at the genetic level, it has not provided a means to differentiate between various sub-types of learning that may occur at this level. Moreover, empirical evidence supporting the idea that genetic material can learn appears tentative, with further research needed to demonstrate that what we are observing are changes in genetic behavior (i.e., changes in how a particular stimulus affects the state of genetic material) that are due to a regularity in the environment. ${ }^{9}$

\footnotetext{
${ }^{8}$ Our example of genetic operant conditioning is similar to work on the operant conditioning of autonomic responses (e.g., heart rate). Debate continues as to whether direct operant conditioning of autonomous responses is possible. Whether this is also true for genetic behavior remains to be seen (for more on this topic see ${ }^{[8]}$ ).

${ }^{9}$ Research on phenomena such as epigenetic priming and transcriptional memory show that earlier events in the environment can influence how the genetic system responds to later events in the environment. This begs the question: is genetic learning just another way of speaking about phenomena such as epigenetic priming or is it something else entirely? From our perspective, these phenomena are different. Epigenetic priming often involves a single exposure to just one stimulus, which then alters gene expression. This does not qualify as genetic learning because genetic learning - by definition - requires that a change in behavior is due to a regularity in the environment. Whereas a regularity always involves more than one stimulus at one point in time, instances of epigenetic priming may already occur after a single stimulus (i.e., the prime). For more details on the relation between priming and learning in general, see Footnote 3 of the introductory chapter of [8].
} 


\section{Genetic Learning (Regularity: two stimuli)}

Time 1: Genetic behavior of interest is absent

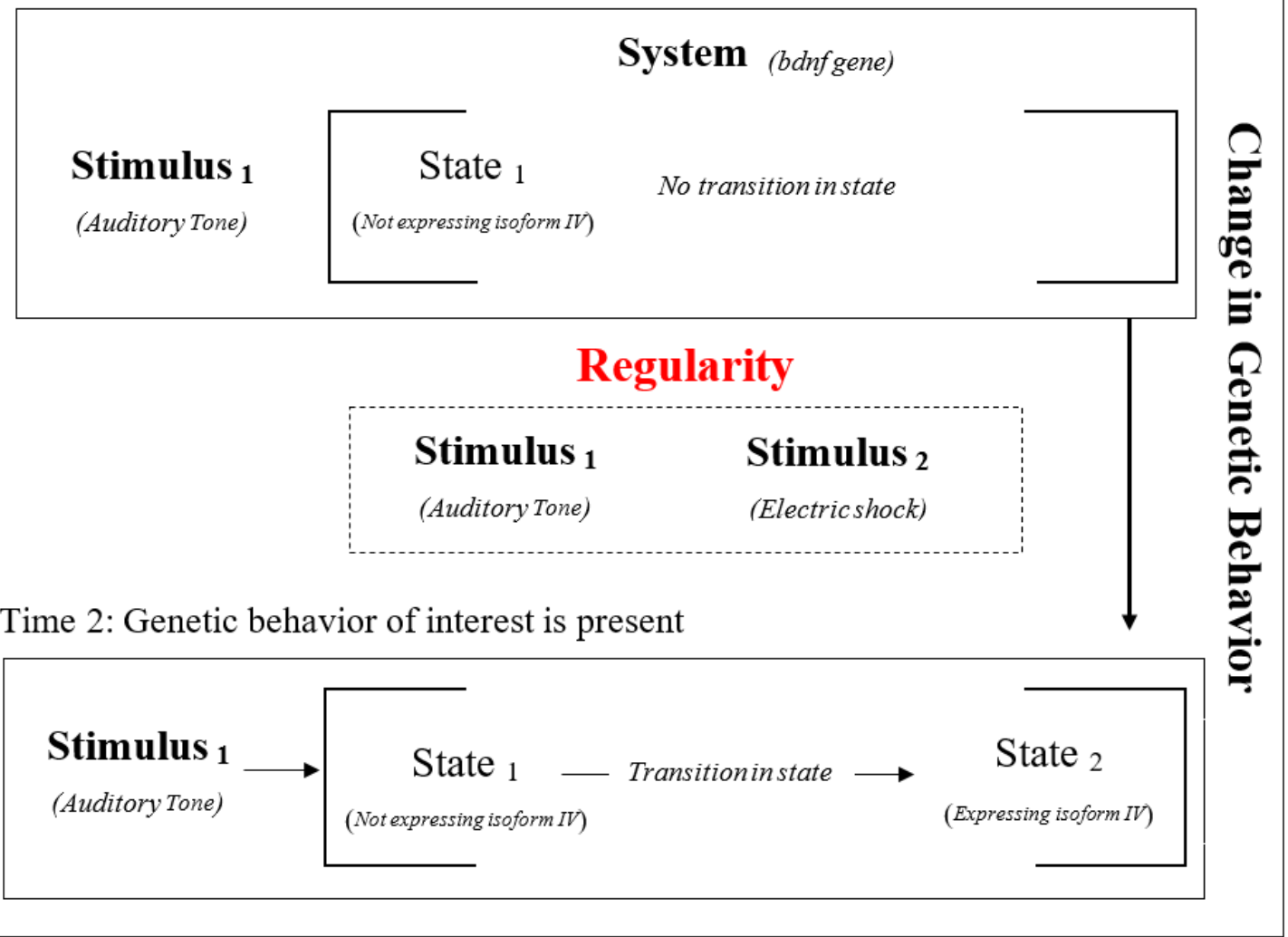

Figure 6. A visual illustration of a change in genetic behavior that is due to a regularity involving two stimuli. At Time 1 being exposed to an auditory tone (Stimulus 1) does not lead to the transition in state we are interested in. Later on we pair the tone (Stimulus 1) with administration of electric shocks (Stimulus 2) either once or multiple times. At Time 2, the tone (Stimulus 1) leads to the transition in state we are interested in (mRNA expression of isoform IV). The increase in mRNA expression from Time 1 to 2 constitutes a change in genetic behavior and this change is due to a regularity between two stimuli (i.e., pairing the tone with electric shocks) that occurred between Time 1 and Time 2. This constitutes a second type of learning known as a genetic classical conditioning effect.

\section{Part III: What are the Merits of Our Approach?}

The ideas outlined in Parts I and II allow us to better organize and understand past work on genetic activity (heuristic value), and introduce interesting new questions about how the genetic system potentially reacts and adapts to the wider environment (generative value).

\section{Heuristic Value}

The approach outlined in this paper argues that there are three types of activity at the genetic level: genetic behavior, changes in genetic behavior, and genetic learning. When studying genetic behavior one will focus on a transition in state of genetic material and seek to explain that transition through reference to a stimulus (e.g., show that exposure to light [stimulus] leads to 'clock gene' expression [transition in state]). Those interested in changes in genetic behavior will simply document differences between transitions in state without reference to a particular cause of that change (e.g., show that ringing a bell does not lead to 'clock gene' expression at Time 1 whereas ringing it at Time 2 does). Finally, those interested in genetic learning will focus on changes in genetic behavior that are due to a regularity in the environment (e.g., show that ringing a bell does not lead to clock gene expression at Time 1, but after the bell is repeatedly paired with light [regularity], ringing the bell at Time 2 does lead to expression).

The above definitions give rise to a new conceptual framework for studying genetic activity, one that allows us to understand what unites different researchers (i.e., their common interest in the activity of the genetic system) as well as the dimensions along which they differ (e.g., one researcher may be interested in explaining genetic behavior while another is interested in genetic learning). By using terms that are both abstract and general this framework accommodates a variety of existing concepts (e.g., transcriptional learning, epigenetic priming, stress memory) and explains how and why such phenomena are similar or 
Genetic Learning (Regularity: stimulus \& response)

Time 1: Gene expressed at a high level
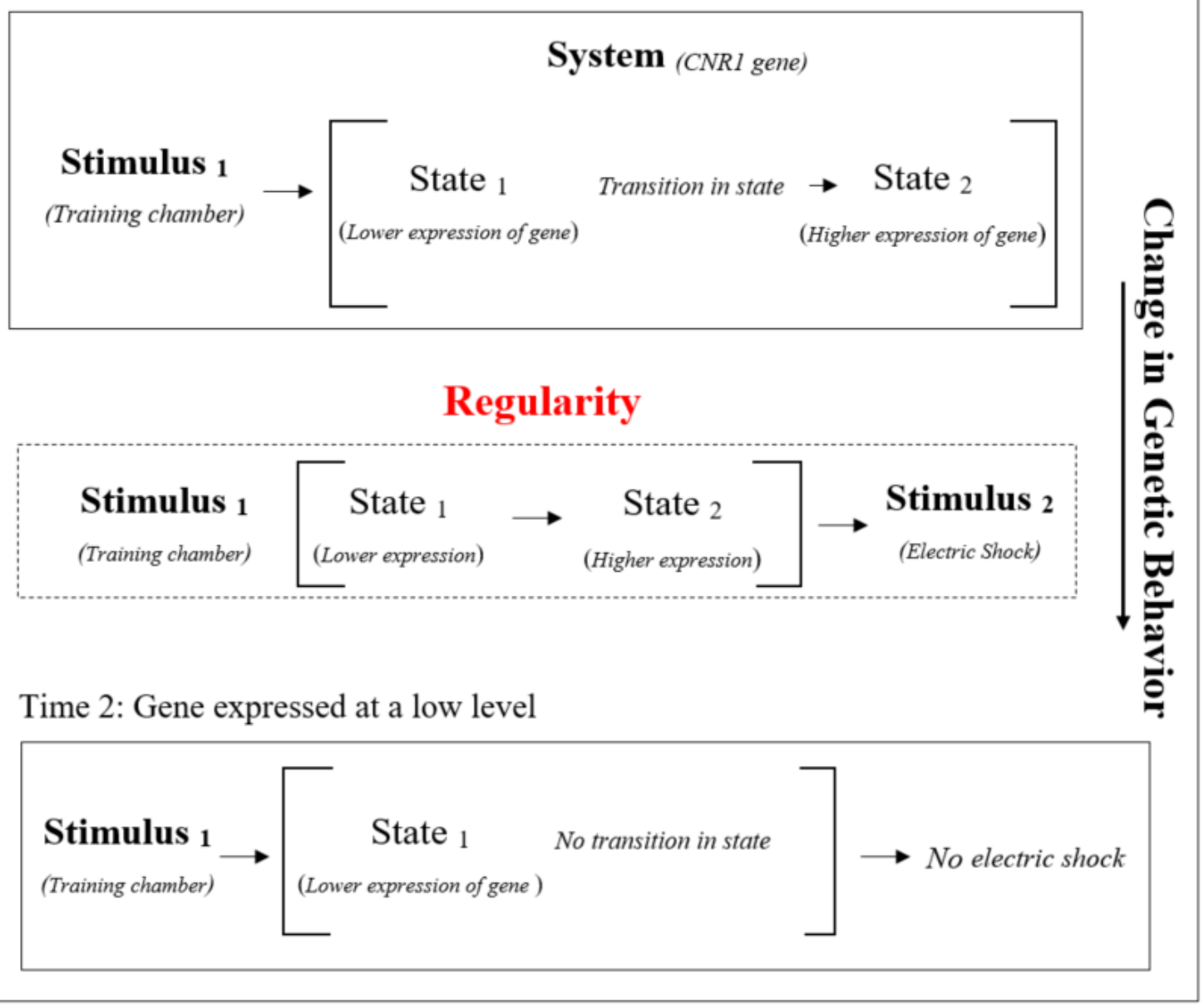

Figure 7. A visual illustration of a change in genetic behavior due to a regularity involving stimuli and responses. At Time 1, placing a rat into a training chamber (Stimulus ${ }_{1}$ ) leads the system (CNR1 gene) to transition from one state to another (to an increase in gene expression). Later on, a regularity is introduced: each time this transition in state occurs (gene expression) inside of the training chamber (Stimulus ${ }_{1}$ ) an electric shock (Stimulus ${ }_{2}$ ) is delivered. At Time 2, the rat is placed back into the training chamber and gene expression is now low. The decrease in the rate of gene expression from Time 1 to 2 constitutes a change in behavior and this change is due to a regularity involving stimuli and responses (i.e., to the fact that gene expression previously led to an electric shock inside the training chamber) that occurred between Time 1 and Time 2. This represents a genetic operant conditioning effect.

different to one another (e.g., how epigenetic priming differs from learning; see Footnote 10).

At the same time, it also provides clear criteria for those looking to study genetic learning. In order to claim that genetic learning has taken place three conditions must be met: (a) some change in genetic behavior needs to be observed, (b) a regularity must be present, and (c) the presence of the former needs to be causally attributed the latter. Yet many studies at this level are correlational in nature: they study associations' between stimuli (e.g., nutritional stress, psychosocial stress, environmental toxins) and genetic expression, and as such, only tell us about how the genetic system behaves (i.e. that the system transitions its state because of a stimulus; [34]). If we want to go one step further, and show that the genetic system learns, we need to put that system into contact with regularities, and show that this leads to a change in genetic behavior. Past work has made use of regularities in the lab (e.g., exposed systems to fear or operant conditioning paradigms) [25-26]. However, because of how these studies were designed (i.e., their focus on the impact of a stimulus on transitions in state), they only tell us that genetic material can behave and do not provide the necessary and sufficient conditions to conclude that it can learn (i.e., that the impact of a stimulus on the transition in state of genetic material differs as a function of a regularity in the environment). 
We believe this is an area ripe for systematic future exploration. ${ }^{10}$

\section{Generative Value}

The approach outlined in this paper also introduces new questions about genetic learning and the conditions under which it occurs. In Part II, for instance, we argued that the genetic system may be capable of learning in three different ways. Future work could examine if our idea has merit (i.e., if it's possible to sensitize, habituate, classically condition, or operant condition genetic material). To do so, one would simply have to assess the behavior of genetic material at Time 1 (i.e., how it reacts to a stimulus [ringing bell]), introduce a regularity (e.g., pairing the stimulus [ringing bell] with another stimulus [electric shock]), and then examine genetic behavior again at Time 2 (i.e., see if gene expression has changed in the presence of the ringing bell). Such work could be carried out using whole organisms (expose them to a regularity while registering the impact that it has on their genetic behavior), or in petri dishes wherein cell assemblies or DNA segments are exposed to substances to see if they learn. Others could examine the factors that moderate genetic learning. Nearly a century's worth of research with whole organisms tells us that learning is moderated by many factors, from the types of regularity, stimuli, responses, contexts, and/or systems involved (see [8]). This may also be true when it comes to the genetic system. Researchers could examine if learning at the genetic level depends on the system involved (it may be that material from different individuals in the same species, or from different species, behaves and learns at different rates in and different ways), or the context it finds itself in (e.g., stimuli and environments 'internal' to the body [presence or absence of certain chemicals inside the cell] may moderate learning in ways that are different to stimuli and environments in the 'external' world [socioeconomic status or cultural practices]). Others could examine if it's easier for the genetic system to learn in one way compared to others (e.g., it may be easier to sensitize or habituate genetic material than classically or operant condition it).

Even this is just the tip of the iceberg. Researchers could examine if learning at the genetic level generalizes from one stimulus, response, or context to another. Or if genetic learning (i.e., the effects of a regularity on genetic behavior) can be counteracted by subsequent experiences (see [40] [41]). Others could explore if genetic learning depends on issues of timing (e.g., are there "windows of susceptibility" during which certain genetic material is particularly sensitive to environmental regularities; see [45]), how persistent those effects are (e.g., are these changes in genetic behavior still evident many years after the regularities have stopped), and if they are heritable across generations (i.e., inter- and transgenerational inheritance of learning effects)? Indeed, it may be that what is learned by, and passed down through, previous generations to the current generation, influences how genetic material behaves and learns (in the same way that evolutionary history has prepared us to enjoy certain [sugary and salty] foods from birth and dislike certain organisms such as snakes and spiders).

The take home message here is that allowing for the idea that genetic material not only behaves but learns, has generative value. Examining if and under which conditions genetic material learns, whether changes in genetic behavior can be counteracted, generalized, inherited, and the numerous other questions outlined above, allows us to better predict how the genetic system reacts to the environment. It also highlights those aspects of the environment that can be used to influence the probability of future genetic activity. This understanding of what moderates genetic learning can also help us to better understand the (neural, biochemical) mechanisms via which the environment influences changes in genetic behavior (e.g., [43] [44]), while mechanistic discoveries at the genetic level can be incorporated into the study of learning in other systems (e.g., whole organisms). In short, we envision a multi-disciplinary field centered on genetic learning research that will help transform our understanding of the way in which genetic material adapts to the environment.

Conclusion

In his book 'The Developing Genome' David Moore suggests that genetic material and "environments are engaged in a kind of conversation that is ongoing throughout our lives. The characteristics we wind up with - from our temperaments to our diseases - emerge from the collaborative actions of both genetic and environmental factors." [2] Exploring the idea of genetic learning allowed us to highlight that genetic material and the environment are not engaged in a conversation but rather different kinds of conversations, and to equip researchers with conceptual tools that help them better understand what exactly nature and nurture are saying

\footnotetext{
${ }^{10}$ Research shows that transitions in states of genetic material can occur as a function of 'environmental events' such as diet and nutrition ${ }^{[35]}[36]$, social status ${ }^{[37]}$, or aversive life experiences ${ }^{[38]}\left[{ }^{[39]}\right.$. Although it may be tempting to view these 'events' as a single stimulus they may involve regularities between stimuli and/or responses, and thus represent instances of genetic learning rather than simple behavior. For instance, one's 'diet' typically involves repeated exposure to the same types of food across time and thus represents a regularity in the occurrence of one stimulus (see ${ }^{[40]}$ for a learning perspective on diets in whole organisms). Adverse life experiences (e.g., poor parenting) involve contact with stimuli that influence the probability of responding (e.g., if a child's crying [response] is followed by physical abuse [stimulus] then crying will likely decrease in frequency) (i.e., a regularity between stimuli and responses). In short, complex environmental events previously studied in this literature may involve regularities rather than single stimuli, and thus involve genetic learning rather than behavior.
} 
to one another. We hope the ideas contained in this paper spark new dialogue between those interested in what exactly nature and nurture are saying to one another.

\section{Notes}

SH and JDH, Department of Experimental Clinical and Health Psychology, Ghent University. The preparation of this paper was supported by Grant BOF16/MET_V/002 of Ghent University to JDH. Correspondence concerning this article should be sent to sean.hughes@ugent.be. Our sincere thanks to Adelheid Soubry for her invaluable input on the paper.

\section{References}

[1] N. Carey. The Epigenetics Revolution: How Modern Biology Is Rewriting our Understanding of Genetics, Disease, and Inheritance. Columbia University Press, New York, NY, 2012.

[2] D. S. Moore. The Developing Genome: An Introduction to Behavioral Epigenetics. Oxford University Press, New York, NY, 2015.

[3] M. L. Holland, R. Lowe, P. W. Caton, C. Gemma, G. Carbajosa, A. F. Danson, A. A. Carpenter, E. Loche, S. E. Ozanne, V. K. Rakyan. Science, 2016, 353, 495.

[4] J. C. Jiménez-Chillarón, R. Díaz, D. Martínez, T. Pentinat, M. Ramón-Krauel, S. Ribó, T. Plösch. Biochimie, 2012, 94, 2242.

[5] D. Mehta, T. Klengel, K. N. Conneely, A. K. Smith, A. Altmann, T. W. Pace, M. Rex-Haffner, A. Loeschner, M. Gonik, K. B. Mercer, B. Bradley. Proc Natl Acad Sci, 2013, 110, 8302.

[6] M. Kundakovic, F. A. Champagne. Neuropsychopharmacol, 2015, 40, 141.

[7] D. A. Hackman, M. J. Farah, M. J. Meaney. Nat. Rev. Neurosci, 2010, 11, 651.

[8] J. De Houwer, S. Hughes, S. The Psychology of Learning: An Introduction from a Functional Cognitive Perspective. The MIT Press, 2020.

[9] A. B. Barron, E. A. Hebets, T. A. Cleland, C. L. Fitzpatrick, M. E. Hauber, J. R. Stevens. Trends Neurosci, 2015, 38, 405.

[10] S. J. Lachman. J. Psychol, 1997, 131, 477.

[11] J. De Houwer, D. Barnes-Holmes, A. Moors. Psychon Bull Rev, 2013, 20,631.

[12] M. B. Gerstein, C. Bruce, J. S. Rozowsky, D. Zheng, J. Du, J. O. Korbel, O. Emanuelsson, Z. D. Zhang, S. Weissman, M. Snyder. Genome Res, 2007, 17, 669.

[13] O. Van Cauwenbergh, A. Di Serafino, J. Tytgat, A. Soubry. Clin. Epigenetics, 2020, 12, 1-23.

[14] T. Wang, C. C. Hung, D. J. Randall. Annu. Rev. Physiol., 2006, 68, 223.

[15] L. Madaniyazi, S. Li, S. Li, Y. Guo. Environ. Int., 2020, 105, 610 .

16] H. González-Pardo, M. P. Álvarez. Psicothema, 2013, 25, 3 .
[17] M. Nishi, N. Horii-Hayashi, T. Sasagawa. Front Neurosci, 2014, 8, 166.

[18] N. Horii-Hayashi, T. Sasagawa, W. Matsunaga, Y. Matsusue, C. Azuma, M. Nishi. J. Neuroendocrinol., 2013, 25, 158.

[19] Y. Ding, M. Fromm, Z. Avramova. Nat. Commun., $2012,3,1$.

[20] L. Virlouvet, M. Fromm. New Phytol, 2015, 205, 596.

[21] Z. Avramova. The Plant Journal, 2015, 83, 149.

[22] A. Chittka, L. Chittka. PLoS Biol, 2010, 8, e1000532.

[23] R. Kucharski, J. Maleszka, S. Foret, R. Maleszka. Science, 2008, 319, 1827.

[24] M. Wojciechowski, R. Lowe, J. Maleszka, D. Conn, R. Maleszka, P. J. Hurd. Genome Res., 2018, 28, 1532 .

[25] F. D. Lubin, T. L. Roth, J. D. Sweatt. J Neurosci, 2008, 28, 10576.

[26] C. A. Miller, J. D. Sweatt. Neuron, 2007, 53, 857.

[27] B. G. Dias, S. A. Maddox, T. Klengel, K. J. Ressler. Trends Neurosci, 2015, 38, 96.

[28] S. Kim, \& B. K. Kaang. Exp Mol Med, 2017, 49, e281-e281.

[29] I.B. Zovkic, J. D. Sweatt. Neuropsychopharmacol, 2013, 38, 77.

[30] S. Mancino, A. B, J. Gutiérrez-Cuesta, M. Gutiérrez-Martos, E. Martín-García, M. Pucci, A. F. C. D'addario, M. Maccarrone, R. Maldonado. Neuropsychopharmacol, 2015, 40, 2788.

[31] F. M. Vassoler, S. L. White, H. D. Schmidt, G. Sadri-Vakili, R. C. Pierce. Nat. Neurosci., 2013, 16, 42.

[32] M. E. Andrzejewski, B. L. McKee, A. E. Baldwin, L. Burns, P. Hernandez, P. Neurosci Biobehav Rev, 2013, 37, 2071.

[33] F. Zoratto, E. Romano, E. Pascale, M. Pucci, A. Falconi, B. Dell'Osso, M. Maccarrone, G. Laviola, C. D'Addario, W. Adriani. Neuroscience, 2017, 340, 101.

[34] Z. M., Thayer C. W. Kuzawa. Epigenetics, 2011, 6, 798 .

[35] S. W. Choi, S. Friso. Adv Nutr, 2010, 1, 8.

[36] P. O. McGowan, M. J. Meaney, M. Szyf. Brain Res., 2008, 1237, 12.

[37] J. Tung, L. B. Barreiro, Z. P. Johnson, K. D. Hansen, V. Michopoulos, D. Toufexis, K. Michelini, M. E. Wilson, Y. Gilad. Proc Natl Acad Sci, 2012, 109, 6490.

[38] P. E. Lutz, G. D. N. A. Turecki. Neuroscience, 2014, 264, 142.

[39] T. L. Roth, F. D. Lubin, A. J. Funk, J. D. Sweatt. Biol. Psychiatry, 2009, 65, 760.

[40] L. H. Epstein, J. L. Temple, J. N. Roemmich, M .E. Bouton. Psychol. Rev, 2009, 116, 384.

[41] K. M. Lattal, M. A. Wood. Nat. Neurosci., 2013, 16, 124 . 
[42] X. Li, Q. Zhao, W. Wei, Q. Lin, C. Magnan, M. R. Emami, L. E. Wearick-Silva, T. W. Viola, P. R. Marshall, J. Yin, S. U. Madugalle. Nature Neuroscience, 2019, 22, 534.

[43] M. E. Bouton. Learning and Behavior: A Modern Synthesis. Sinauer, Sunderland, MA 2016.
[44] M. A. Gluck, E. Mercado, C. E. Myers. Learning and Memory: From Brain to Behavior. Worth Publishers, New York, NY, 2016. 